\title{
Stress-mediated hyperactivity and anhedonia resistant to diazepam and fluoxetine in Drosophila
}

\section{Ana Belén Ramos-Hryb, Mauro Federico Ramirez, Cilene Lino-de-Oliveira \& Mario Rafael Pagani}

To cite this article: Ana Belén Ramos-Hryb, Mauro Federico Ramirez, Cilene Lino-de-Oliveira \& Mario Rafael Pagani (2020): Stress-mediated hyperactivity and anhedonia resistant to diazepam and fluoxetine in Drosophila, Stress

To link to this article: https://doi.org/10.1080/10253890.2020.1759547

Accepted author version posted online: 22 Apr 2020.

Submit your article to this journal $₫$

Q View related articles ¿

View Crossmark data $[\pi$ 
Title: Stress-mediated hyperactivity and anhedonia resistant to diazepam and fluoxetine in Drosophila

\section{Running title: Short variable stress effects in flies}

Authors: Ramos-Hryb, Ana Belén ${ }^{1,2 \&}$, Ramirez, Mauro Federico ${ }^{1}$, Lino-de-Oliveira, Cilene ${ }^{2,3}$, Pagani, Mario Rafael ${ }^{1 *}$

\section{Affiliations:}

${ }_{1}$ Instituto de Fisiología y Biofísica (IFIBIO) Bernardo Houssay, Grupo de Neurociencia de Sistemas, Universidad de Buenos Aires, CONICET, Buenos Aires, Argentina.

${ }_{2}$ Postgraduation Program in Pharmacology, CCB, Federal University of Santa Catarina, Florianópolis, Brazil.

${ }_{3}$ Department of Physiological Sciences, CCB, Federal University of Santa Catarina, Florianópolis, Brazil.

${ }_{\&}$ Current Address: Institute of Biology and Experimental Medicine (IBYME)-CONICET, Buenos Aires, Argentina.

\section{*Corresponding author:}

Dr. Mario Rafael Pagani, E-mail: pagani@fmed.uba.ar, calciumsignal@gmail.com

Word Count: 6388 words 


\begin{abstract}
:
Distresses may induce behavioral phenotypes constituting heuristic models for psychopharmacology studies. In several species, including Drosophila, antidepressants counteract stress-induced phenotypes allowing the use of these models to test new psychoactive drugs. Here, we developed a novel and time-efficient protocol to provoke stress-induced phenotypes in Drosophila for the study of psychopharmacological agents. In the first experiment, flies ( $n=12 /$ groups) were exposed to a random-sequence of different types of stresses during nearly $24 \mathrm{~h}$ (including social isolation, fasting, heat, and electric shock), a protocol named short-term variable stress (SVS). Second, flies were exposed to a single stressful stimulus (social isolation, fasting, heat shock or electric shock, $\mathrm{n}=12$ /groups). Next, flies submitted to SVS protocol were treated with vehicle, diazepam or fluoxetine ( $n=12$ /groups). At the end of the stress protocols, behavioral phenotypes were evaluated in the open field (OF) and sucrose preference tests. In comparison to the unstressed group, flies exposed to SVS exhibited hyperactivity, as well as shorter times exploring the boundaries of the OF. In contrast to fasting stress, SVS reduced sucrose preference in flies. By analyzing the effects of individual stimuli on fly behavior, fasting and electric shock appear to be the predominant influences on the SVS-induced behaviors. Although fluoxetine or diazepam reduced the initial locomotor activity of flies, no treatment prevented the sequelae of SVS. Altogether, this study provides a timeefficient model system for the study of stress-mediated hyperactivity and anhedonia-like state resistant to fluoxetine and diazepam. The applications of SVS in Drosophila to preclinical psychopharmacology require further studies.
\end{abstract}

Keywords: preclinical test; chronic unpredictable stress; psychopharmacology; antidepressants; anxiolytics, Drosophila.

\title{
Lay summary
}

Exposition to unpredictable stress plays a significant role in psychiatric disorder's onset. Behavioral traits of these disorders can be partially modeled in rodents aimed at developing psychopharmacological therapies. However, studies in rodents were questioned by ethical issues. Focused on 3Rs principles, we developed a preclinical model for stress and psychopharmacology research in Drosophila. Variable stress induced behavioral alterations, including hyperlocomotion and reduced preference for sucrose in flies. However, behavioral alterations were resistant to fluoxetine and diazepam. 


\section{Introduction}

Distress is an important factor altering animal behavior. Behavioral sequelae of stress are a function of intensity, length, frequency, and predictability of the stimuli (Shors and Servatius, 1997). Animals exposed to variable stresses exhibit learned helplessness or discontinuing attempts to avoid aversive stimuli (Katz et al., 1981; López-López et al., 2016; Mineur et al., 2006; Zhu et al., 2014), and anhedonic-like states or reduced interest for palatable stimuli (López-López et al., 2016; Mineur et al., 2006; Zhu et al., 2014). Anhedonia, despair, and helplessness are symptoms presented in patients with depression (American Psychiatric Association, 2013). Along with this behavioral phenotype, rodents show neurochemical alterations also detected in patients, including serotonin (5HT) dysfunctions (Amat et al., 2005; Vollmayr and Gass, 2013).

Stress-mediated behavioral sequelae in rodents are often ameliorated by classical psychopharmacological agents, including fluoxetine and diazepam (Giacomini et al., 2016; Gupta et al., 2014). Reversion of these phenotypes can be used to predict the antidepressant activity of novel pharmacological agents (Porsolt et al., 1977; Willner, 2017). However, rodent testing received criticism and questioning in the field of ethics and animal welfare (Reardon, 2019). In fact, several initiatives are in place to increase the validity of behavioral tests, based on the ethical principles of animal experimentation such as refining, reducing and replacement (3Rs) (Graham and Prescott, 2015). Focusing on these principles, this work aimed to design a novel animal model of stressmediated behavior alterations in Drosophila.

Drosophila is a suitable organism for modeling human diseases due to their phylogenetic relationship with vertebrates and powerful genetic tools (Bellen et al., 2010). Moreover, monoamines are required to control fly behaviors (Hidalgo et al., 2017; Mohammad et al., 2016a; Neckameyer and Matsuo, 2008; Ries et al., 2017). The evolutionary conserved role of 5-HT in stress-dependent behaviors, reinforces the idea of using Drosophila in neurobiology research (Kasture et al., 2018; Ries et al., 2017). Few attempts to develop a model for depression in Drosophila have been made since Brown et al (1996) reported learned helplessness in flies (Brown et al., 1996). Noteworthy flies chronically exposed to vibrations reduced walking or climbing as compared to controls. This depressive-like state correlated with reduced 5-HT release at the mushroom body which was reverted by the serotonin precursor 5-hydroxy-L-tryptophan (Ries et al., 2017). In addition, Drosophila exposed for ten days to random sequences of variable stresses exhibited high immobility, aggressiveness, and anhedonia-like behavior, all reverted by fluoxetine (Araujo et al., 2018). Thus far, no study reported a short, random and variable protocol that induces stress phenotypes for rapid 
screening of psychopharmacological agents. The aim of this study was to develop a novel and timeefficient stress protocol for the study of psychopharmacological agents in Drosophila.

\section{Methods}

\section{Animals}

Adult male and female wild-type Canton-S (RRID: BDSC_64349) Drosophila melanogaster (from 2 to 6 days after pupae eclosion) were used. Canton-S strands were obtained from Bloomington Drosophila Stock Center and maintained in $37 \mathrm{ml}$ polystyrene vials containing $10 \mathrm{ml}$ of standard corn medium (Pagani et al., 2009). Until the experiment, flies were maintained with a mean number of 50 flies per vial under controlled conditions at $25{ }^{\circ} \mathrm{C}, 55-65 \%$ humidity and 12:12 h darklight cycle (lights on at 8 a.m.). In this study, 12 flies were used per treatment and group and all of the animals were included in the analysis.

\section{Drugs}

Fluoxetine (Eli Lilly, Indianápolis, U.S.A.) and Diazepam (LaboratoriosFabra, Buenos Aires, Argentina) were diluted at the final concentrations of 10 and $5 \mathrm{mM}$, respectively. These concentrations were based on previous studies with flies showing that $10 \mathrm{mM}$ fluoxetine treatment suppressed the altered behavior induced by a vibration-stress protocol (Ries et al., 2017), $5 \mathrm{mM}$ of diazepam counteracted the anxiety-like state and hyperlocomotion (Mohammad et al., 2016a) and 10 $\mu \mathrm{M}$ ketamine promoted neuritogenesis and synaptogenesis in Drosophila larvae (Ly et al., 2018). Flies were transferred to an empty vial containing a filter paper $(3 \times 3 \mathrm{~cm})$ soaked with $120 \mu 1$ of either vehicle (water), $10 \mathrm{mM}$ fluoxetine, $5 \mathrm{mM}$ diazepam or $10 \mu \mathrm{M}$ ketamine and kept there for 6 hours (during the entire period of fasting). We chose this time period because a previous study showed that 4 hours of fasting is sufficient to induce a food-seeking behavior in flies (Root et al., 2011). Sucrose obtained from Anedra (AN00711812, Buenos Aires, Argentina) prepared at the final concentration of $2 \mathrm{M}$ was used for the sucrose preference test. This concentration was based on a study that demonstrated that fasted flies exhibit a higher sucrose preference within the first 20-30 s of exposition to this solution (Thoma et al., 2016). In order to have the same time gap between drug treatments and the behavioral tests, we used a specific arrangement in the presentation of stimuli. Fasting was always applied as a second factor and it was followed either by heat (20 min) and electric shock $(5 \mathrm{~min})$ or electric $(5 \mathrm{~min})$ and heat shock stresses $(20 \mathrm{~min})$. Therefore, the time gap between drug treatments and the behavioral tests always corresponded to $25 \mathrm{~min}$. All drugs were diluted in distilled water. 


\section{Stress protocols}

Setting up a short protocol of stress was the first step to establish a system for testing and examining the mechanisms of psychopharmacological drugs in flies. The selection of types of stress to be applied to the flies was performed after a series of pilot studies. The main criteria to select the type of stress were: 1- feasibility in the laboratory conditions; 2- avoidance of extremely aggressive stimuli; and 3-time-efficiency of the test performing. Hence the following stimuli were selected: social isolation $(18 \mathrm{~h})$, heat shock $(20 \mathrm{~min})$, fasting $(6 \mathrm{~h})$ and electric shock (5 min). The protocols of stress consisted of a single session (monotonic stress) or a combination of different types of stress randomly presented without a rest period in between. This last protocol was named short-term variable stress (SVS). In addition, monotonic stress groups were used to assess whether SVS-induced behaviors resulted from the combination of the stressful stimuli or, predominantly, by one or more stimuli.

\section{Stress stimuli}

For social isolation stress, adult flies were individually transferred into polystyrene $37 \mathrm{ml}$ vials containing standard corn medium for 24 hours at $25{ }^{\circ} \mathrm{C}$ and $55-65 \%$ of humidity (Pagani et al., 2009). For heat shock stress, 4-5 flies were introduced into previously heated glass-tubes of $20 \mathrm{~mm}$ diameter and $50 \mathrm{~mm}$ length containing a filter paper to avoid water condensation inside the tube, and immersed into a water bath at $37{ }^{\circ} \mathrm{C}$ during $20 \mathrm{~min}$. This treatment was based on pilot experiments showing that this condition was the minimal time of exposition able to alter the locomotory behavior of flies. For fasting stress, flies were individually transferred into an empty $37 \mathrm{ml}$ vial containing a paper soaked with water for preventing dehydration. Fasting was performed for 6 hours. To induce electric shock stress, 4-5 flies were transferred into a tube with electric grids placed internally over the walls, where 60 volts were delivered for $5 \mathrm{~min}$ in $1.5 \mathrm{~s}$ bouts with $5 \mathrm{~s}$ of resting intervals (Tully et al., 1994). For each experiment, the control condition corresponded to a group of animals transferred to fresh food vials containing standard corn medium under controlled conditions $\left(120 \mathrm{Lux}, 25^{\circ} \mathrm{C}\right.$, $55-65 \%$ except for electric shock where the humidity was controlled and set to $70 \%$ ) which is called as "control naïve group".

\section{SVS protocol}

Groups of adult flies were exposed to a specific, randomly distributed combination of social isolation (18 h), heat shock (20 min), fasting (6 h) and electric shock (5 min) stress without resting intervals between stimuli (Figure 1). Exposition to SVS protocol was conducted in an artificially illuminated room (120 Lux) under constant environmental conditions $\left(25^{\circ} \mathrm{C}\right.$, 55-65\% except for 
electric shock where the humidity was controlled and set to $70 \%$ ). The control naïve group remained in a 50 flies-population and it was transferred to fresh food $37 \mathrm{ml}$ vials with standard corn medium under controlled conditions $\left(25^{\circ} \mathrm{C}, 55-65 \%\right)$. Behavioral experiments were conducted between 11:00 am to 7:00 pm. Flies were transferred into the behavioral arenas immediately after finishing the stress exposition. The time of the day, the sex of animal or the age of animals used did not affect locomotion (Figure S3-5).

\section{Behavioral tests}

In order to quantify the behavior performance of individual flies, a behavioral box was designed and constructed. The behavioral box consisted of a black box of $30 \times 10 \times 10 \mathrm{~cm}$ in height, width and depth of foamed polyvinyl chloride ( $3 \mathrm{~mm}$ of thickness). The box was divided into three sections (lower, medium, and upper compartments) by two shelves of white acrylic at a height of 10 and 20 $\mathrm{cm}$, respectively (Figure S1). The lower and upper compartments of the box contained a white lightemitting diode light source that illuminated the lower and upper faces of the arena (240 lux on the top and 80 lux on the bottom) through a white acrylic diffuser. The medium compartment contained a semicircular indentation that allowed placing the experimental arena always in the same position. The upper compartment, with a $2 \times 2 \mathrm{~cm}$ window, supported a high definition camera Logitech HD Pro Webcam C910. Thus, OF and sucrose preference tests were video recorded from above.

\section{OF Test}

Circular plastic Petri dishes of $58 \mathrm{~mm}$ in diameter and $10 \mathrm{~mm}$ in height were used for the OF test. The walls of the arena were covered with an alternate black and white pattern. The roof of the arena was a circular plastic lid of petri dish of $8.8 \mathrm{~mm}$ in diameter with a perforation of $1 \mathrm{~cm}$ in diameter next to its edges that allowed the entry of animals into the arena. Flies were transferred into the arena and tested for 10 min. Arenas were cleaned with $70 \%$ alcohol and leave to dry between tests to minimize any pheromonal or waste products that might affect the behavior of the next tested flies.

\section{Sucrose preference test}

Fruit flies were tested for sucrose preference using a circular arena (with identical dimension and configuration with the OF arena). Each half of the arena was covered with a semicircular piece of filter paper that had been soaked with either $250 \mu \mathrm{l}$ of water or $250 \mu \mathrm{l}$ of a $2 \mathrm{M}$ sucrose solution as previously described (Thoma et al., 2016). Then, flies were allowed to choose between the two sides for $1 \mathrm{~min}$. Arenas were cleaned with water followed by $70 \%$ alcohol and leave to dry between tests. 


\section{Behavioral recording and analysis}

Once the flies were transferred into the arena, the behavior was immediately video recorded with a high definition camera at $30 \mathrm{~Hz}$ using VirtualDub (v 1.10.4, Avery Lee). The videos were converted into 320x240 photograms with Prism Video Converter Software (v 3.04, NCH Software) and analyzed using ANY-Maze ${ }^{\circledR}$ Video Tracking System (Stoelting Co.). For the OF test the following variables were analyzed: habituation index, total distance traveled during the 10-min session divided by 1-min blocks, total mobility and immobility times (considering immobility if the animal does not change location and if $65 \%$ of its body remains static for at least $3000 \mathrm{~ms}$ ), freezing time (considering freezing if the animal remains at least $500 \mathrm{~ms}$ with a threshold between 30 and 40 scores), freezing and immobility latencies, mean velocity and time expended in borders and/or center of the arena. Habituation index was calculated as follows: (distance traveled during the $1^{\text {st }}$ min distance traveled during the $10^{\text {th }} \mathrm{min}$ )/(distance traveled during the $1^{\text {st }} \mathrm{min}+$ distance traveled during the $10^{\text {th }} \mathrm{min}$ ). The sucrose preference index was calculated as (time on sugar - time on water)/(time on sugar + time on water). The border versus center index was measured as (time on border)/(time on center).

\section{Statistical tests}

Statistical analysis was carried out using Graph pad Prism v. 7.04 (California Corporation) and StatSoft v. 7.0 (Tulsa, U.S.A.). Normal distribution and homogeneity of variances were checked using the Shapiro-Wilk test and Bartlett's test, respectively. Comparison between two unpaired groups was conducted by one-sided student's t-test or Mann Whitney (M-W) and, the analysis between two paired groups was performed with Wilcoxon Matched Pairs Test. Comparison between more than two groups was conducted by one-way analysis of variances (ANOVAs) followed by Newman Keuls's (N-K) post-hoc. When data were not normally distributed, comparison between more than two groups was conducted by Kruskal Wallis (K-W) followed by Dunn post-hoc. Differences with a probability of $5 \%$ or less were considered to be statistically significant. Sample size estimation for a statistical power of $80 \%$ was conducted using G*Power. Outliers were identified using the Grubbs' test-based calculator where $\alpha=0.05$. The following criteria were set beforehand to exclude outliers: values derived from tracking errors in the Any-maze analysis, errors of data entry or values scientifically impossible (e.g. negative distance traveled). The number of outliers identified per group may be seen in Table $\mathbf{S 1}$. 


\section{Results}

\section{SVS induces hyperlocomotion and centrophilic behavior in adult flies}

To establish an efficient model system in fruit flies that allow us to test traditional and novel psychopharmacological drugs, we began by setting up a short protocol of stress. We designed a protocol of acute and variable stress that combines several types of stress randomly without a rest period in between. This protocol consists of a single combination of the following stimuli: social isolation (18 h), heat shock (20 min), fasting (6 h) and electric shock (5 min) (Figure 1). We named this new protocol SVS, (see section: SVS protocol).

To assess the stress-induced phenotypes of the short-term SVS protocol in the locomotor behavior, flies were evaluated in the OF test after exposure to stress. The temporal analysis in oneminute time segments revealed no statistical difference between curves of exploration of control naïve and stressed groups (K-W test between control naïve and stressed groups of minute $1: \mathrm{H}_{(1,24)}=$ 1.92, $\mathrm{p}>0.05$; minute $2: \mathrm{H}_{(1,24)}=0.96, \mathrm{p}>0.05$; minute $3: \mathrm{H}_{(1,24)}=0.85, \mathrm{p}>0.05$; minute $4: \mathrm{H}_{(1,24)}=$ 0.70, $\mathrm{p}>0.05$; minute $5: \mathrm{H}_{(1,24)}=3.00, \mathrm{p}>0.05$; minute $6: \mathrm{H}_{(1,24)}=1.92, \mathrm{p}>0.05$; minute $7: \mathrm{H}_{(1,24)}=$ 2.25, $\mathrm{p}>0.05$; minute 8: $\mathrm{H}_{(1,24)}=1.84, \mathrm{p}>0.05$; minute 9: $\mathrm{H}_{(1,24)}=0.48, \mathrm{p}>0.05$; minute $10: \mathrm{H}_{(1,24)}=$ 2.90, p>0.05; Figure 2A). Flies in control naïve (Wilcoxon matched pairs test, $t=8.00, p<0.05$ ) and stressed (Wilcoxon matched pairs test, $\mathrm{t}=10.00, \mathrm{p}<0.05$ ) groups showed higher exploratory activity in the beginning compared with the end of the test (Figure 2B). Although stressed flies exhibited a less steep reduction $(56 \%)$ in the traveled distance parameter between the final and initial minutes when compared to control naïve flies (80\%), no significant difference in the habituation index was seen (Table S2). Total distance traveled (one-tailed unpaired student's t-test; $t_{(22)}=3.31, p<0.01$, Figure 2C) and the mean velocity (one-tailed unpaired student's $t$-test; $t_{(22)}=3.31, p<0.01$, Figure 2D) were higher in the animals exposed to the SVS protocol compared with the control naïve group. Accordingly, total immobility time of stressed flies was lower compared with control naïve flies (one-tailed unpaired student's t-test; $\mathrm{t}_{(22)}=2.31, \mathrm{p}<0.05$, Figure $2 \mathbf{E}$ ). Stressed flies also showed a lower preference for the border of the OF compared with control naïve group (one-tailed unpaired $\mathrm{M}-\mathrm{W}, \mathrm{U}=29.50, \mathrm{p}<0.01$, Figure 2F). Whereas control naïve flies spent almost $81 \%$ of the testing time within the outermost zone of the arena, stressed flies spent only $39 \%$ of the testing time in this region. Finally, the SVS-protocol did not affect significantly the immobility latency and the total freezing time in flies (Table S2). 


\section{Behavioral phenotype of flies exposed to individual stressful stimuli}

Variable, mild stresses may produce more complex behavioral phenotypes than a single stressful stimulus (Katz et al., 1981; López-López et al., 2016; Mineur et al., 2003, 2006; Zhu et al., 2014). However, it is not clear which is the contribution of each individual stressful stimulus to the SVSmediated phenotypes reported above (Figure 2). To further characterize this protocol and understand the interaction between stimuli, we examined the effects of presenting each stressful stimulus separately. Exposure to twenty-four hours of isolation did not alter the habituation index, the total distance, the mean velocity, the total immobility time or the preference for the border or center of the arena in flies (Figure S2, Table S2). In contrast, all the other stressful stimuli presented individually produced a stress-depended behavioral phenotype (Figure 3). Compared to the non-stressed group, flies exposed to twenty minutes of heat shock covered less distance during the testing time (onetailed unpaired M-W; U=27, p<0.01, Figure 3A) and explored the OF with lower mean velocity (one-tailed unpaired $\mathrm{M}-\mathrm{W}, \mathrm{U}=31, \mathrm{p}<0.01$, Figure 3B). Heat shock also increased the total time of immobility during the OF test (one-tailed unpaired $\mathrm{M}-\mathrm{W} ; \mathrm{U}=22, \mathrm{p}<0.01$, Figure 3C) without changing the preference of flies to the border of the apparatus (one-tailed unpaired $M-W ; U=65$, p $>0.05$ Figure 3D to E). In contrast, exposure of flies to 6 hours of fasting increased the distance traveled (one-tailed unpaired student's t-test; $t_{(22)}=4.06, p<0.001$, Figure 3F), the mean velocity (one-tailed unpaired student's t-test; $\mathrm{t}_{(22)}=4.04, \mathrm{p}<0.001$, Figure 3G), reduced the immobility time (one-tailed unpaired student's t-test; $\mathrm{t}_{(22)}=2.58, \mathrm{p}<0.01$, Figure $3 \mathbf{H}$ ) and increased the exploration of the center of arena (one-tailed unpaired student's t-test; $\mathrm{t}_{(22)}=3.84, \mathrm{p}<0.001$, Figure 3I to $\mathbf{J}$ ) when compared with control naïve flies. Similarly, exposure to $5 \mathrm{~min}$ of electric shocks increased the total distance (one-tailed unpaired student's t-test; $\mathrm{t}_{(22)}=2.38, \mathrm{p}<0.05$, Figure $3 \mathbf{K}$ ) and the mean velocity (one-tailed unpaired student's $\mathrm{t}$-test; $\mathrm{t}_{(22)}=2.81, \mathrm{p}<0.01$, Figure $\mathbf{3 L}$ ) of flies during the OF test. Different from fasting, electric shocks failed to affect immobility time (one-tailed unpaired student's t-test; $\mathrm{t}_{(22)}=0.31, \mathrm{p}>0.05$, Figure $3 \mathbf{M}$ ) and border/center ratio (one-tailed unpaired student's t-test; $\mathrm{t}_{(22)}=0.28, \mathrm{p}>0.05$, Figure $3 \mathbf{N}$ and $\left.\mathbf{O}\right)$.

\section{SVS attenuates the sucrose preference in adult flies}

To investigate the influence of the SVS protocol on anhedonic-like behaviors, immediately after the OF test, flies were exposed for $60 \mathrm{~s}$ to a sucrose preference test. It is well established that flies fed ad libitum shows no preference for sucrose respect to water in a behavioral test, whereas fasted flies do (Root et al., 2011). Thus, since the SVS protocol contained $6 \mathrm{~h}$ of fasting, we compared the sucrose preference of flies fed ad libitum, flies fasted for $6 \mathrm{~h}$ and stressed flies. As expected, 
differences in the preference index between fed, fasted and stressed flies were statistically significant (One-way ANOVA, $\mathrm{F}_{(2,33)}=23.87 ; \mathrm{p}<0.0001$ ). The forward post-hoc analysis revealed that fasted (One-way ANOVA with N-K, $\mathrm{F}_{(2,33)}=23.87$; $\mathrm{p}<0.0001$ ) and stressed (One-way ANOVA with N-K, $\left.\mathrm{F}_{(2,33)}=23.87 ; \mathrm{p}<0.05\right)$ flies exhibited a significantly higher preference for sucrose compared with animals fed ad libitum (Figure 4). However, the SVS protocol reduced the sucrose preference induced by fasting (One-way ANOVA with $\mathrm{N}-\mathrm{K}, \mathrm{F}_{(2,33)}=23.87$; $\mathrm{p}<0.001$; Figure 4D).

Acute treatment with diazepam or fluoxetine failed to counteract the hyperactivity and border/center preference induced by SVS in the OF

In the last experiment, we examined whether diazepam or fluoxetine treatments counteract the behavioral phenotype of flies exposed to the SVS protocol. The temporal analysis of the locomotor behavior of flies from the minutes 2 to 10 revealed no statistical differences (K-W ANOVA test between flies treated with vehicle, diazepam or fluoxetine of minute $2: \mathrm{H}_{(2,36)}=3.39, \mathrm{p}>0.05$; minute 3: $\mathrm{H}_{(2,36)}=2.72, \mathrm{p}>0.05$; minute $4: \mathrm{H}_{(2,36)}=2.94, \mathrm{p}>0.05$; minute $5: \mathrm{H}_{(2,36)}=0.96, \mathrm{p}>0.05$; minute 6 : $\mathrm{H}_{(2,36)}=2.09, \mathrm{p}>0.05$; minute 7: $\mathrm{H}_{(2,36)}=2.64, \mathrm{p}>0.05$; minute $8: \mathrm{H}_{(2,36)}=1.17$, $\mathrm{p}>0.05$; minute 9: $\mathrm{H}_{(2}$, $36)=2.43, \mathrm{p}>0.05 ;$ minute $10: \mathrm{H}_{(2,36)}=0.59, \mathrm{p}>0.05$; Figure 5A). However, diazepam or fluoxetine significantly reduced the total distance traveled during the first $60 \mathrm{~s}$ compared with vehicle (K-W ANOVA between flies treated with vehicle, diazepam or fluoxetine of minute $1: \mathrm{H}_{(2,36)}=6.38$, $\mathrm{p}<0.05$, Figure 5A). In addition, distance traveled in the last minute of the OF test was lower than the observed in the initial minute in flies treated with vehicle (Wilcoxon matched pairs test, $\mathrm{t}=7.00$, $\mathrm{p}<0.05$ ), diazepam (Wilcoxon matched pairs test, $\mathrm{t}=5.00, \mathrm{p}<0.05$ ) or fluoxetine (Wilcoxon matched pairs test, $\mathrm{t}=13.00, \mathrm{p}<0.05)$. However, neither diazepam nor fluoxetine significantly altered the habituation index, immobility latency or the total freezing time (Table S2). In addition, total distance $(\mathrm{K}-\mathrm{W}=1.66$, $\mathrm{p}>0.05$, Figure $5 \mathrm{C})$, the mean velocity $(\mathrm{K}-\mathrm{W}=2.66, \mathrm{p}>0.05$, Figure 5D) and total immobility time (One-way ANOVA, $\mathrm{F}_{(2,33)}=0.04$; $\mathrm{p}>0.05$, Figure 5E) were similar between groups. Finally, neither diazepam nor fluoxetine altered significantly the time expended in the periphery of the $\mathrm{OF}$ arena $(\mathrm{K}-\mathrm{W}=0.17, \mathrm{p}>0.05$, Figure $5 \mathrm{~F})$. 


\section{The treatment of flies with diazepam or fluoxetine failed to counteract the SVS-reduced preference for sucrose}

Finally, we asked whether treatment with diazepam or fluoxetine would reverse the low preference for sucrose in stressed animals. None of the pharmacological treatments reverted the reduced preference for sucrose induced by the SVS protocol (One-way ANOVA, $F_{(2,33)}=0.21$; $\mathrm{p}>0.05$, Figure 6). However, as figure 6D shows, diazepam significantly changed the data distribution ( $\mathrm{p}=0.03$, Kolmogorov-Smirnov) as well as the median ( $\mathrm{U}=34 ; \mathrm{p}=0.02$, Mann-Whitney) compared with vehicle. Additionally, we asked if fast-acting antidepressant drugs, such as ketamine (Kadriu et al., 2019, Ly et al., 2018) could reverse the locomotory alteration induced by the SVS protocol. However, we did not find significant differences in the total distance traveled, the velocity, the total immobility time, the border versus center zones permanence and sucrose preference index of male and female flies (Figure S6).

\section{Discussion}

Previous studies reported protocols that induce depressive and/or anxiety-like behavior in Drosophila (Araujo et al., 2018; Brown et al., 1996; Ries et al., 2017; Yang et al., 2013). All these studies required a long-time exposition and evaluated later responses of flies to stress. The protocol here presented includes different sources of stress, allows acquiring quick results for drug screening and it is easy to apply in laboratories. Moreover, the randomness order of stimuli applied between individuals as well as during the protocol is an important translational value that reflects the different types of stress present in human life and the individual variability of animal's responses to stress. Finally, the short duration of this protocol allows exploring mechanisms of the early response to stress.

The SVS-induced hyperactivity diverges from the general inactivity reported previously in Drosophila (Araujo et al., 2018; Ries et al., 2017) suggesting that different mechanisms might be involved in this response. This interpretation is also supported by the fact that, in previous reports, the stress-depended phenotypes were ameliorated by increasing the serotonergic tone. In addition, behavioral responses of flies exposed to the variable component of the SVS protocol disagree from the reported in the no-idleness learning paradigm, where yoked flies reduced their locomotor activity instead of increasing it (Batsching et al., 2016; Yang et al., 2013). These differences in the behavioral response may be related to the type, duration, and level of each stress stimulus as well as the timing of locomotor evaluation in this study and, presumably the mechanisms involved in this and previous reports. Indeed, as in vertebrates, acute stress modifies the basal locomotor behavior of 
adult flies and the dopaminergic system have been involved in these locomotor alterations (Argue and Neckameyer, 2013; Chadha and Cook, 2014). In agreement, some studies showed that short (1 hour) (Katz et al., 1981) or long (between 3 to 8 weeks) (Faraji et al., 2014; Harris, 1997) exposition to stress induces enhanced locomotor and exploratory behavior in rats. These findings in rats support the interpretation that the stress-depended hyperactivity and anhedonic-like behavior reported here is a possible behavioral outcome, but not a specific response of invertebrates.

Hyperlocomotion and agitation are key emotional characteristics of mania and, in some cases, of depressive disorders (Mineur et al., 2006; Young et al., 2011). In this regard, the hypothesis was that treatment with diazepam, a classical anxiolytic drug that prevents mania and anxiogenic-like behavior in rodents (Mohammad et al., 2016b), could be also able to revert the hyperlocomotion of flies (Mohammad et al., 2016a). However, in our assays diazepam only reduced the stressindependent locomotor activity and therefore, this protocol failed to show a predictive value. The resistance to diazepam could be due to the short time of the treatment duration, considering the extensive duration of treatments required to revert the anxiogenic-like behavior in rodents (Mohammad et al., 2016b). Several studies have also reported that, according to the preclinical test used, some class of pharmacological agents such as benzodiazepines or serotonergic-related ones, may elicit a mild or even no response in animals (Belovicova et al., 2017; Bourin and Hascoët, 2003; Ennaceur and Chazot, 2016), which may be the case in this study. Besides, diazepam is a fast and long-lasting facilitator of the GABAergic system, which functions as a positive allosteric modulator of $\mathrm{GABA}_{\mathrm{A}}$ receptors via the benzodiazepine receptor (Griffin et al., 2013). The different pharmacological effects elicited by diazepam (anxiolytic, sedative, muscle relaxant, anticonvulsant and amnestic) may depend upon the sensitivity and binding in the benzodiazepine binding site of different types of benzodiazepine-sensitive $\mathrm{GABA}_{\mathrm{A}}$ receptors (containing $\alpha 1, \alpha 2, \alpha 3$ or $\alpha 5$ subunits) (Griffin et al., 2013). More importantly, different types of stressors in rodents may modulate different GABA receptor classes altering binding, sensitivity and response to benzodiazepines (Skilbeck et al., 2010). Hence, it is also possible that the lack of response of flies to diazepam may be accounted by the differential modulation of GABA receptors upon SVS stress.

Fluoxetine is one of the most potent and selective serotonin reuptake inhibitors antidepressants that, upon assessing of the serotonin transporter, inhibits serotonin uptake (Whentur et al., 2013). Specifically, it has strong affinity for $5-\mathrm{HT} 2_{\mathrm{A}}$ and $5-\mathrm{HT} 2_{\mathrm{C}}$ receptor subtypes and quite absent activity at adrenergic, muscarinic, opiate, dopamine, GABA, and histamine receptors. This effect is followed by increases on serotonin concentration with decrease in the synthesis and release of serotonin in several brain regions of rodents (Whentur et al., 2013). Despite this effect occurs immediately, the amelioration of depressive symptoms of patients and rodent's phenotype takes 
more than 4 to 6 weeks (Whentur et al., 2013). In fact, we also observed a resistant-response to fluoxetine upon the SVS protocol, which could be also due to the insufficient time and/or dose required to promote antidepressive-like effects. This long-time period required for the efficacy of fluoxetine may involve several neural adaptations such as protein kinases modulation, production and release of neurotrophins, and modulation of plasma glutamate concentrations (Whentur et al., 2013).

The initial treatment resistance in vertebrate and invertebrates may be because initially, main neurochemical alterations are not monoaminergic or GABAergic. In this regard, rapid-acting antidepressant drugs, such as ketamine, primarily acts as antagonist of NMDA receptors (Kadriu et al., 2019). We tested the hypothesis that a fast-acting antidepressant could ameliorate the behavioral alteration induced by SVS (Kadriu et al., 2019) and we observed that, in our conditions, ketamine was not able to reverse these effects.

One of the interesting findings in this study was the extended time of exploration in the center of the arena produced by the SVS protocol. It is well established that non-stressed flies exhibit a higher preference to explore the boundary of an OF (Besson and Martin, 2005; Götz and Biesinger, 1985; Lebreton and Martin, 2009; Soibam et al., 2012) and that this anxiogenic-like behavior can be reverted with diazepam (Mohammad et al., 2016a). However, the center directed locomotion induced by the SVS protocol was not reverted with diazepam. This behavior is also contrasting with the border directed locomotion of mammals exposed to stress (Harris, 1997), which has been long characterized as an anxiogenic trait (Treit and Fundytus, 1988; Walz et al., 2016). The behavioral pattern observed in this study may be explained, at least partially, by the increased velocity and distance traveled by stressed flies, however further studies are necessary to reveal the mechanism involved in this behavior.

Another interesting behavioral observation is that, despite SVS protocol increased locomotion, the stresses flies showed a reduced activity at the end of the test compared with the initial activity. Therefore, indicating that SVS was not able to alter the habituation index in flies.

In agreement with previous studies, this work shows that exposition of flies only to heat shock reduces the locomotor activity, but fasting and electric shock increases the motor behavior (Bell et al., 1985; Mohammad et al., 2016a; Ostrowski et al., 2018). However, this study contradicts previous reports which showed that isolation reduced the exploratory behavior of adult flies (Araujo et al., 2018; Neckameyer and Nieto-Romero, 2015). Another intriguing result from this study is that the exposition of flies only to fasting inhibits thigmotaxis, which may be related to suppression by synaptic output from mushroom body neurons (Besson and Martin, 2005). Moreover, the inhibition of thigmotaxis induced by SVS may be explained by the fact that fasting increases exploratory and 
food-seeking behavior (Bell et al., 1985; Chadha and Cook, 2014; Soibam et al., 2012) which, in turn, reduces boundary preferences in flies. Therefore, the analysis of individual stress stimulus revealed that fasting and electric shock could be promoting centrophilia and increased locomotion observed in the SVS. It remains to be determined whether a protocol combining only fasting and electric shock is also able to produce the same behavioral phenotype, allowing to obtain an even more time-efficient protocol.

Reduced preference for palatable food, such as sucrose solutions, has been used as a preclinical test of anhedonia in flies (Araujo et al., 2018), a typical symptom of depression (American Psychiatric Association, 2013). Interestingly, the depressive-like state of stressed flies can be reverted by sucrose (Ries et al., 2017). In our study, the SVS protocol reduced the preference for sucrose if compared with fasted flies, suggesting that it precluded the food-seeking behavior induced by fasting. However, it is no clear from this assay if flies actually consumed sucrose. Further studies are required to elucidate whether the SVS-related responses in the preference test indicate an anhedonia-like phenotype induced by the variable stress stimuli or a methodological artifact.

Finally, treatment with diazepam or fluoxetine of SVS-exposed flies only reduced the initial locomotor behavior in the OF without interfering with the later activity. This reduction may imply that both treatments were able to reduce the motivation of flies to explore a novel environment while remaining intact stress-induced behaviors.

In conclusion, here we present a novel and time-efficient protocol of variable stress for adult flies. This novel protocol induces hyperlocomotion, centrophilic and anhedonic-like behavior. Overall, this report provides a quick protocol for preclinical test of psychopharmacological studies in the early response to stress, which is resistant to diazepam and fluoxetine.

\section{Geolocation information}

All the procedures in the study were conducted in the Instituto de Fisiología y Biofísica (IFIBIO) Bernardo Houssay, Grupo de Neurociencia de Sistemas, at Universidad de Buenos Aires, CONICET, Buenos Aires, Argentina.

\section{Acknowledgments}

The authors thank Graciela Ortega for technical support as well as Lorena Rela and Axel Gorostiza for their insightful discussions regarding protocols and results. The authors also thank BioRender for providing images for Figure 1. 


\section{Disclosure statement}

The authors declare no known conflict of interest.

\section{Data availability statement}

As specified in the text, our protocols and data were registered on Open Science Framework (osf.io/6nefq) where the following files are available: complete statistical data and protocols for Anymaze analysis.

\section{Funding details}

This work was supported by CNPq-Proex (Brazil) and CONICET (Argentina).

\section{Notes on contributors}

Ramos-Hryb, A. B.: prepared the study design and protocols; collected, analyzed and discussed data, wrote the manuscript, approved the final version.

Ramirez, M. F.: collected and discussed data, wrote the manuseript, approved the final version.

Lino-de-Oliveira, C.: selected research theme; prepared the study design and protocols; discussed data, wrote the manuscript, approved the final version.

Pagani, M.R.: selected research theme; prepared the study design and protocols; collected, analyzed and discussed data, wrote the manuscript, approved the final version. 


\section{References}

Amat, J., Baratta, M. V., Paul, E., Bland, S.T., Watkins, L.R., Maier, S.F., 2005. Medial prefrontal cortex determines how stressor controllability affects behavior and dorsal raphe nucleus. Nat. Neurosci. 8, 365-371.

American Psychiatric Association, 2013. Diagnostic and statistical manual of mental disorders, 5th Edition. American Psychiatric Publishing, Inc.

Araujo, S.M., Poetini, M.R., Bortolotto, V.C., de Freitas Couto, S., Pinheiro, F.C., Meichtry, L.B., de Almeida, F.P., Santos Musachio, E.A., de Paula, M.T., Prigol, M., 2018. Chronic unpredictable mild stress-induced depressive-like behavior and dysregulation of brain levels of biogenic amines in Drosophila melanogaster. Behav. Brain Res. 351, 104-113.

Argue, K.J., Neckameyer, W.S., 2013. Sexually dimorphic recruitment of dopamine neurons into the stress response circuitry. Behav. Neurosci. 127, 734-743.

Batsching, S., Wolf, R., Heisenberg, M., 2016. Inescapable stress changes walking behavior in flies learned helplessness revisited. PLoS One 11, e0167066.

Bell, W.J., Cathy, T., Roggero, R.J., Kipp, L.R., Tobin, T.R., 1985. Sucrose-stimulated searching behaviour of Drosophila melanogaster in a uniform habitat: modulation by period of deprivation. Anim. Behav. 33, 436-448.

Bellen, H.J., Tong, C., Tsuda, H., 2010. 100 years of Drosophila research and its impact on vertebrate neuroscience: a history lesson for the future. Nat. Rev. Neurosci. 11, 514-522.

Belovicova, K., Bogi, E., Csatlosova, K., Dubovicky, M., 2017. Animal tests for anxiety-like and depression-like behavior in rats. Interdiscip. Toxicol. 10(1), 40-43.

Besson, M., Martin, J.R., 2005. Centrophobism/thigmotaxis, a new role for the mushroom bodies in Drosophila. J. Neurobiol. 62, 386-396.

Brown, G.E., Mitchell, A.L., Peercy, A.M., Robertson, C.L., 1996. Learned helplessness in Drosophila Melanogaster? Psychol. Rep. 78, 962-962.

Bourin, M., Hascoët, M., 2003. The mouse light/dark box test. Eur. J. Pharmacol. 463(1-3), 55-65.

Chadha, A., Cook, B., 2014. The effect of stress on motor function in Drosophila. PLoS One 9, e112076.

Ennaceur, A., Chazot, P.L., 2016. Preclinical animal anxiety research - flaws and prejudices. Pharmacol. Res. Perspect. 4(2), e00223.

Faraji, J., Soltanpour, N., Jafari, S.Y., Moeeini, R., Pakdel, S., Moharreri, A., Metz, G.A.S., 2014. Stress inhibits psychomotor performance differently in simple and complex open field environments. Horm. Behav. 65, 66-75. 
Giacomini, A.C.V.V., Abreu, M.S., Giacomini, L. V., Siebel, A.M., Zimerman, F.F., Rambo, C.L., Mocelin, R., Bonan, C.D., Piato, A.L., Barcellos, L.J.G., 2016. Fluoxetine and diazepam acutely modulate stress induced-behavior. Behav. Brain Res. 296, 301-310.

Götz, K.G., Biesinger, R., 1985. Centrophobism in Drosophila melanogaster. J. Comp. Physiol. A 156, 329-337.

Graham, M.L., Prescott, M.J., 2015. The multifactorial role of the 3Rs in shifting the harm-benefit analysis in animal models of disease. Eur. J. Pharmacol. 759, 19-29.

Griffin, C.E. 3rd, Kaye, A.M., Bueno, F.R., Kaye, A.D. 2013. Benzodiazepine pharmacology and central nervous system-mediated effects. Ochsner J.13(2), 214-223.

Gupta, D., Radhakrishnan, M., Kurhe, Y., 2014. 5HT3 receptor antagonist (ondansetron) reverses depressive behavior evoked by chronic unpredictable stress in mice: Modulation of hypothalamic-pituitary-adrenocortical and brain serotonergic system. Pharmacol. Biochem. Behav. 124, 129-136.

Harris, R., 1997. Failure to change exploration or saccharin preference in rats exposed to chronic mild stress. Physiol. Behav. 63, 91-100.

Hidalgo, S., Molina-Mateo, D., Escobedo, P., Zárate, R. V., Fritz, E., Fierro, A., Perez, E.G., Iturriaga-Vasquez, P., Reyes-Parada, M., Varas, R., Fuenzalida-Uribe, N., Campusano, J.M., 2017. Characterization of a novel Drosophila SERT mutant: insights on the contribution of the serotonin neural system to behaviors. ACS Chem. Neurosci. 8, 2168-2179.

Kadriu, B., Musazzi, L., Henter, I.D., Graves, M., Popoli, M., Zarate, C.A., 2019. Glutamatergic neurotransmission: pathway to developing novel rapid-acting antidepressant treatments. Int. J. Neuropsychopharmacol. 22(2), 119-135

Kasture, A., Hummel, T., Sucic, S., Freissmuth, M., 2018. Big lessons from tiny flies: Drosophila melanogaster as a model to explore dysfunction of dopaminergic and serotonergic neurotransmitter systems. Int. J. Mol. Sci. 19, 1788.

Katz, R.J., Roth, K.A., Carroll, B.J., 1981. Acute and chronic stress effects on open field activity in the rat: Implications for a model of depression. Neurosci. Biobehav. Rev. 5, 247-251.

Lebreton, S., Martin, J.-R., 2009. Mutations affecting the cAMP transduction pathway disrupt the centrophobism behavior. J. Neurogenet. 23, 225-234.

López-López, A.L., Bonilla, H.J., Escobar Villanueva, M. del C., Brianza, M.P., Vázquez, G.P., Alarcón, F.J.A., 2016. Chronic unpredictable mild stress generates oxidative stress and systemic inflammation in rats. Physiol. Behav. 161, 15-23.

Ly, C., Greb, A.C., Cameron, L.P., Wong, J.M., Barragan, E.V., Wilson, P.C., Burbach, K.F., Soltanzadeh Zarandi, S., Sood, A., Paddy, M.R., Duim, W.C., Dennis, M.Y., McAllister, A.K., 
Ori-McKenney, K.M., Gray, J.A., Olson, D.E.2018. Psychedelics Promote Structural and Functional Neural Plasticity. Cell Rep. 23, 3170-3182.

Mineur, Y.S., Belzung, C., Crusio, W.E., 2006. Effects of unpredictable chronic mild stress on anxiety and depression-like behavior in mice. Behav. Brain Res. 175, 43-50.

Mineur, Y.S., Prasol, D.J., Belzung, C., Crusio, W.E., 2003. Agonistic behavior and unpredictable chronic mild stress in mice. Behav. Genet. 33, 513-519.

Mohammad, F., Aryal, S., Ho, J., Stewart, J.C., Norman, N.A., Tan, T.L., Eisaka, A., ClaridgeChang, A., 2016a. Ancient anxiety pathways influence Drosophila defense behaviors. Curr. Biol. 26, 981-986.

Mohammad, F., Ho, J., Woo, J.H., Lim, C.L., Poon, D.J.J., Lamba, B., Claridge-Chang, A., $2016 b$. Concordance and incongruence in preclinical anxiety models: Systematic review and metaanalyses. Neurosci. Biobehav. Rev. 68, 504-529.

Neckameyer, W.S., Matsuo, H., 2008. Distinct neural circuits reflect sex, sexual maturity, and reproductive status in response to stress in Drosophila melanogaster. Neuroscience 156, 841856.

Neckameyer, W.S., R. Nieto-Romero, A., 2015. Response to stress in Drosophila is mediated by gender, age and stress paradigm. Stress 18, 254-266.

Ostrowski, D., Salari, A., Zars, M., Zars, T., 2018. A biphasic locomotor response to acute unsignaledhigh temperature exposure in Drosophila. PLoS One 13, e0198702.

Pagani, M.R., Oishi, K., Gelb, B.D., Zhong, Y., 2009. The phosphatase shp2 regulates the spacing effect for long-term memory induction. Cell 139, 186-198.

Porsolt, R.D., Bertin, A., Jalfre, M., 1977. Behavioral despair in mice: a primary screening test for antidepressants. Arch. Int. Pharmacodyn. Ther. 229, 327-36.

Reardon, S., 2019. Depression researchers rethink popular mouse swim tests. Nature 571, 456-457.

Ries, A.-S., Hermanns, T., Poeck, B., Strauss, R., 2017. Serotonin modulates a depression-like state in Drosophila responsive to lithium treatment. Nat. Commun. 8, 15738.

Root, C.M., Ko, K.I., Jafari, A., Wang, J.W., 2011. Presynaptic facilitation by neuropeptide signaling mediates odor-driven food search. Cell 145, 133-144.

Shors, T.J., Servatius, R.J., 1997. The contribution of stressor intensity, duration, and context to the stress-induced facilitation of associative learning. Neurobiol. Learn. Mem. 68, 92-96.

Skilbeck, K.J., Johnston, G.A., Hinton, T. 2010. Stress and GABA receptors. J. Neurochem.112(5), 1115-1130. 
Soibam, B., Mann, M., Liu, L., Tran, J., Lobaina, M., Kang, Y.Y., Gunaratne, G.H., Pletcher, S., Roman, G., 2012. Open-field arena boundary is a primary object of exploration for Drosophila. Brain Behav. 2, 97-108.

Thoma, V., Knapek, S., Arai, S., Hartl, M., Kohsaka, H., Sirigrivatanawong, P., Abe, A., Hashimoto, K., Tanimoto, H., 2016. Functional dissociation in sweet taste receptor neurons between and within taste organs of Drosophila. Nat. Commun. 7, 10678.

Treit, D., Fundytus, M., 1988. Thigmotaxis as a test for anxiolytic activity in rats. Pharmacol. Biochem. Behav. 31, 959-962.

Tully, T., Preat, T., Boynton, S.C., Del Vecchio, M., 1994. Genetic dissection of consolidated memory in Drosophila. Cell 79, 35-47.

Vollmayr, B., Gass, P., 2013. Learned helplessness: unique features and translational value of a cognitive depression model. Cell Tissue Res. 354, 171-178.

Walz, N., Mühlberger, A., Pauli, P., 2016. A human open field test reveals thigmotaxis related to agoraphobic fear. Biol. Psychiatry. 80, 390-397.

Wenthur, C.J., Bennett, M.R., Lindsley, C.W. 2013. Classics in Chemical Neuroscience: Fluoxetine (Prozac). ACS Chem.Neurosci.5, 14-23.

Willner, P., 2017. The chronic mild stress (CMS) model of depression: History, evaluation and usage. Neurobiol. Stress 6, 78-93.

Yang, Z., Bertolucci, F., Wolf, R., Heisenberg, M., 2013. Flies cope with uncontrollable stress by learned helplessness. Curr. Biol. 23, 799-803.

Young, J.W., Henry, B.L., Geyer, M.A., 2011. Predictive animal models of mania: hits, misses and future directions. Br. J. Pharmacol. 164, 1263-1284.

Zhu, S., Shi, R., Wang, J., Wang, J.F., Li, X.M., 2014. Unpredictable chronic mild stress not chronic restraint stress induces depressive behaviours in mice. Neuroreport 25, 1151-1155. 

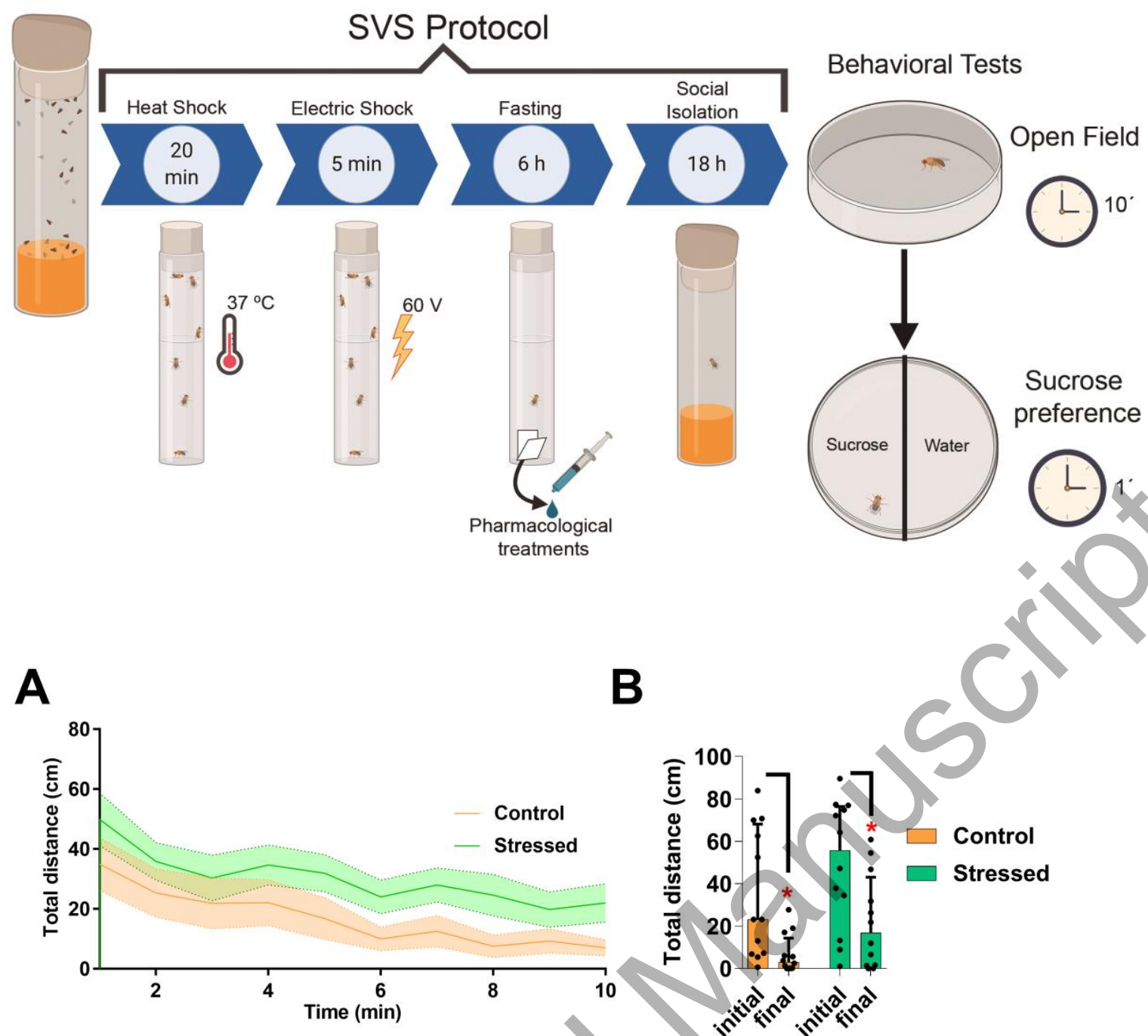

B

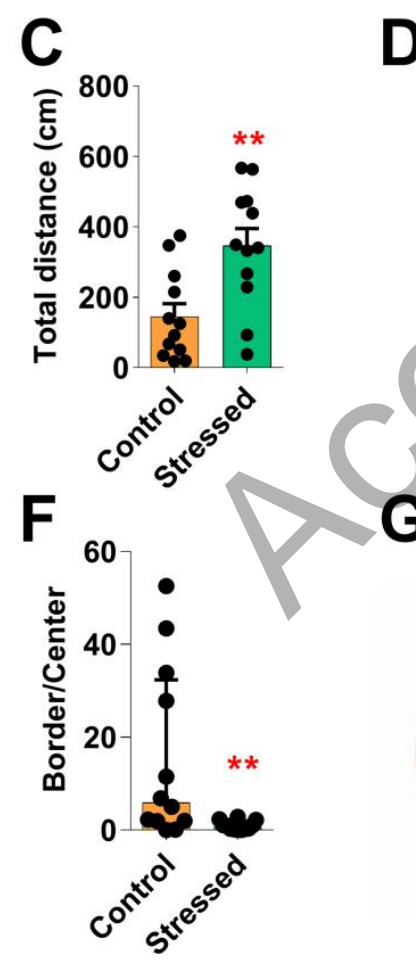

D
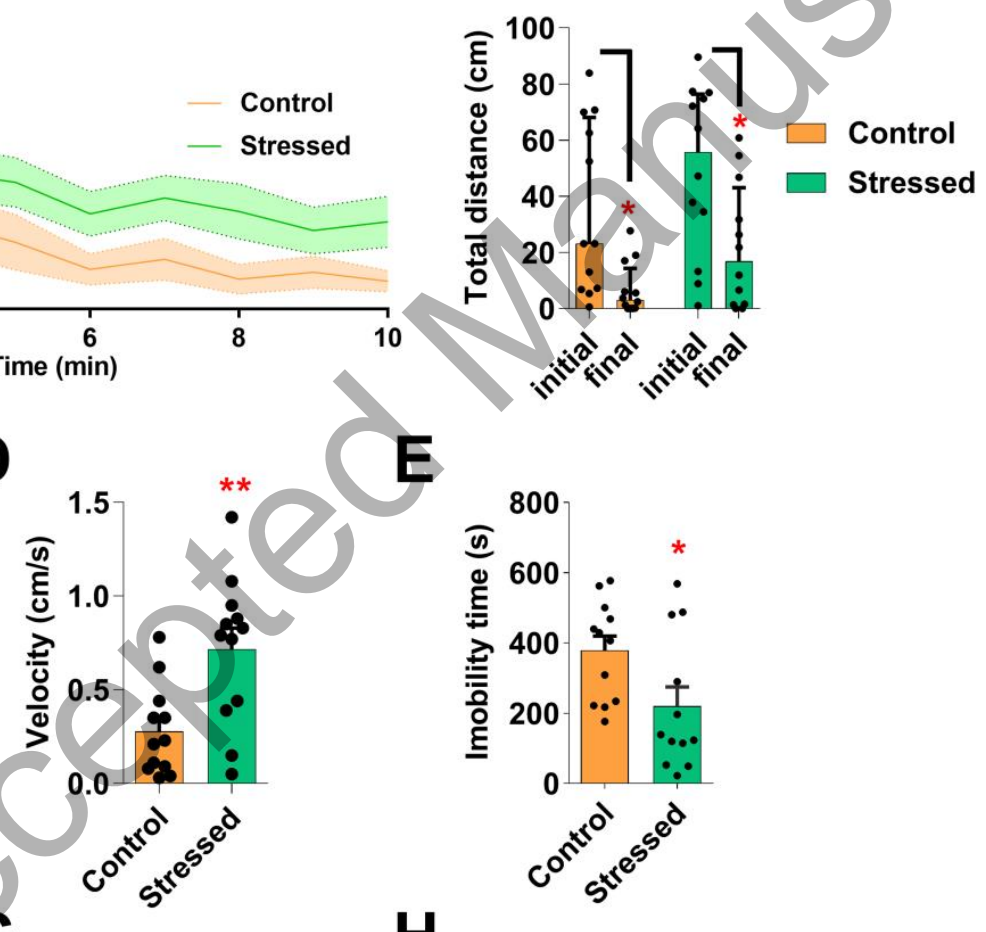

$\mathbf{G}$

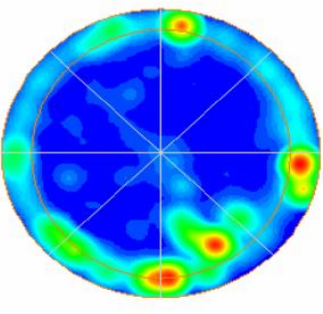

Control

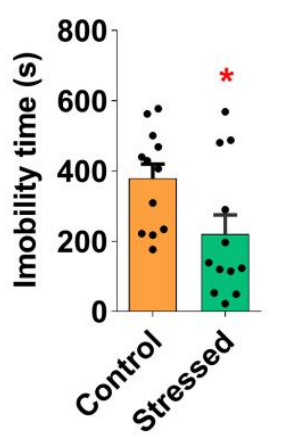

H

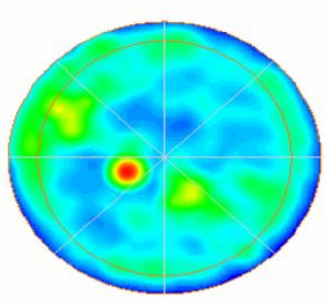

Stressed 


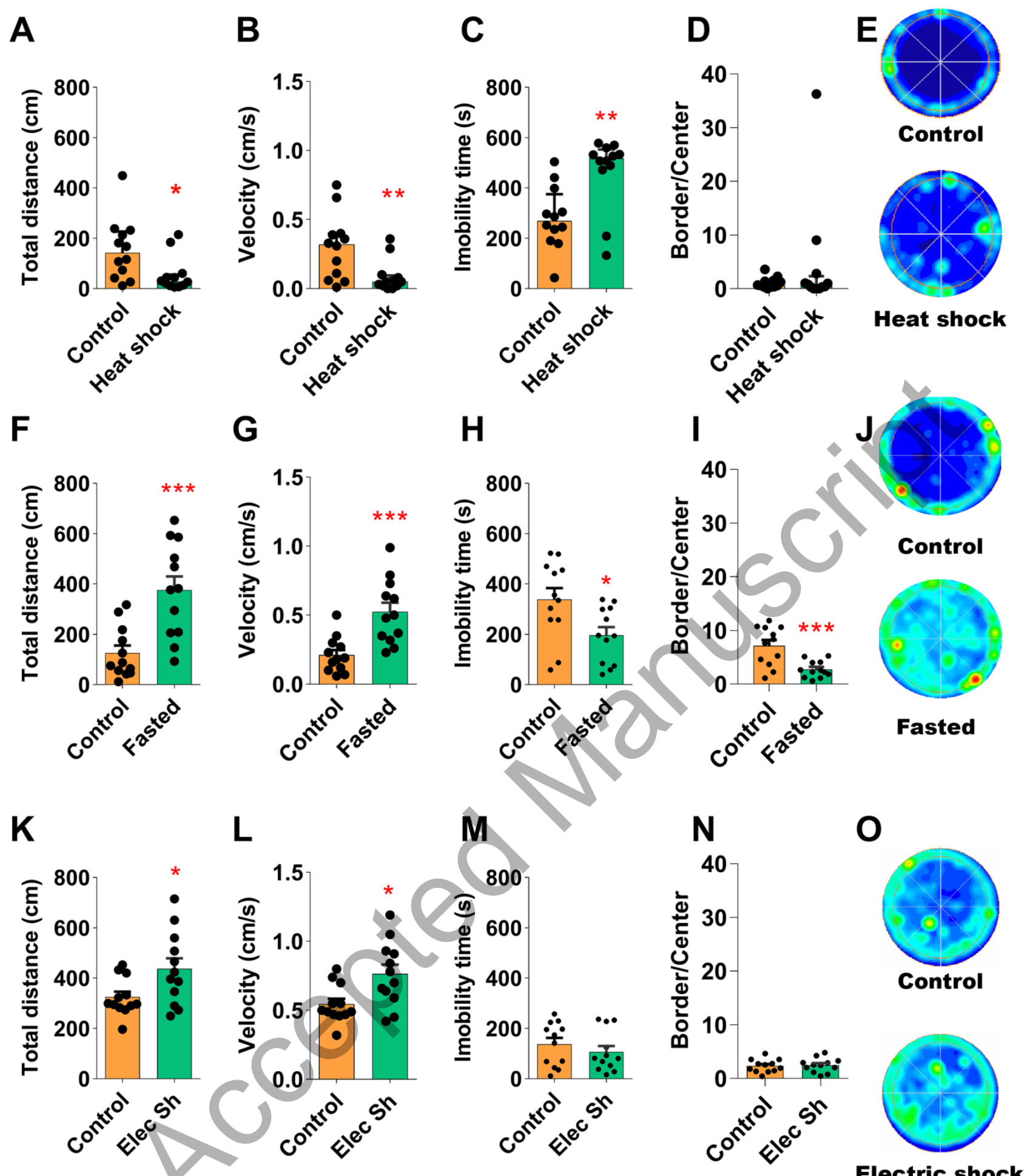




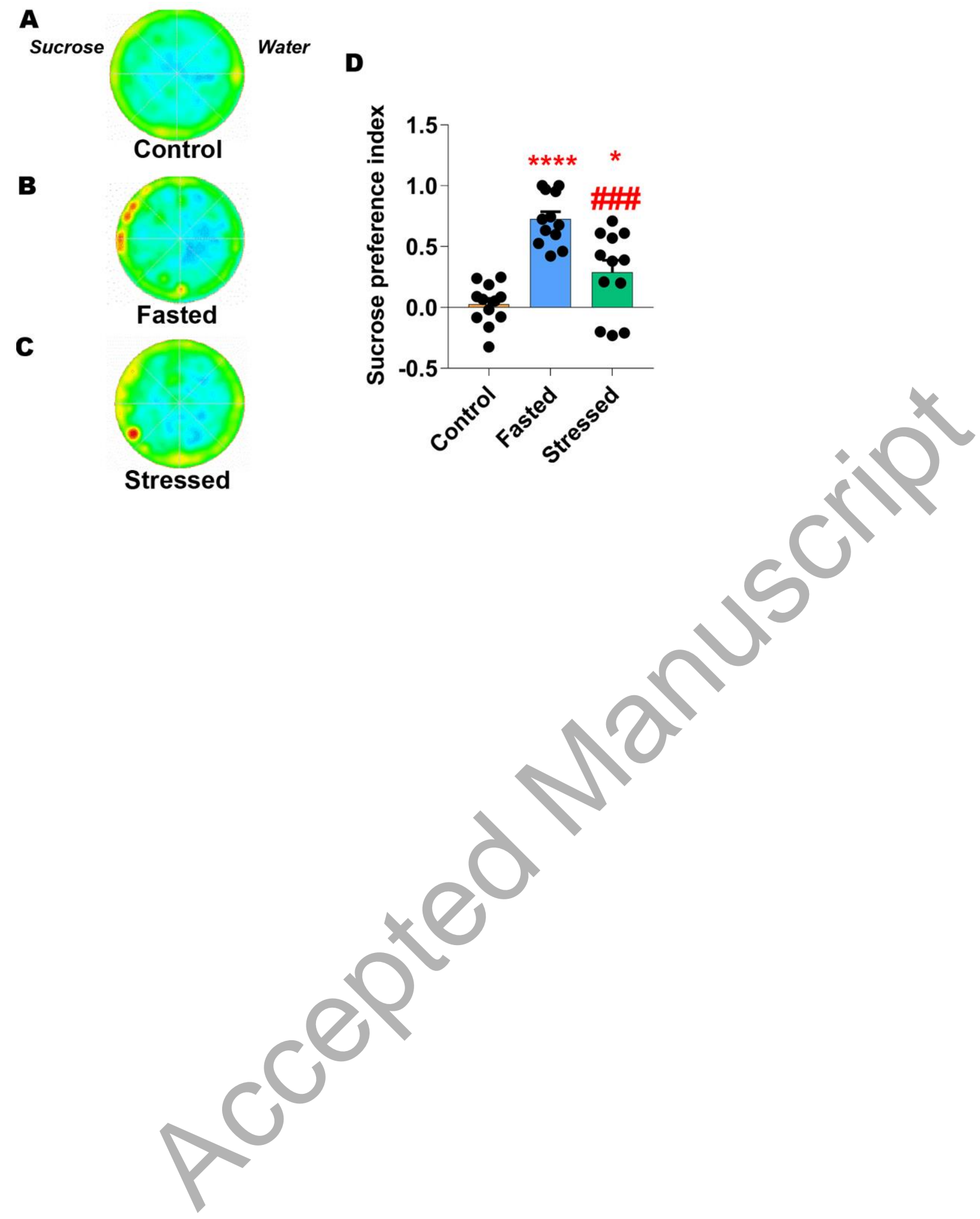


A

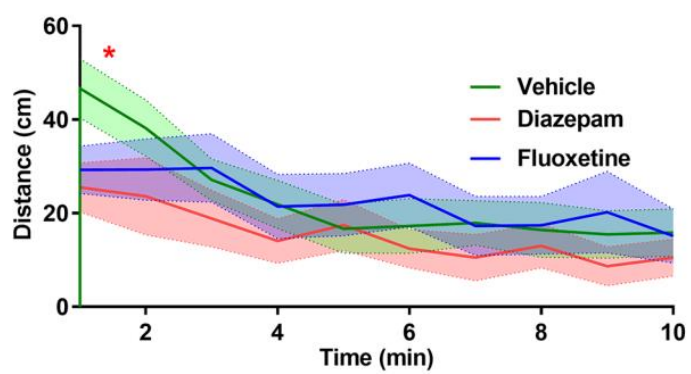

C

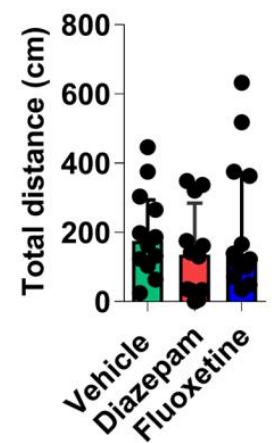

G

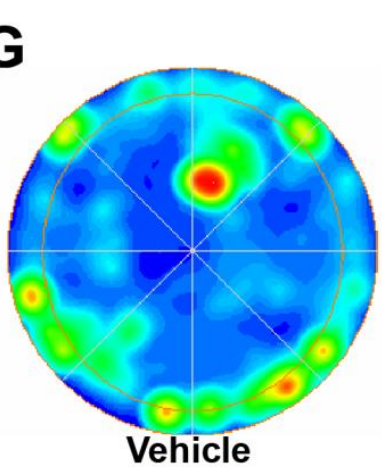

Vehicle

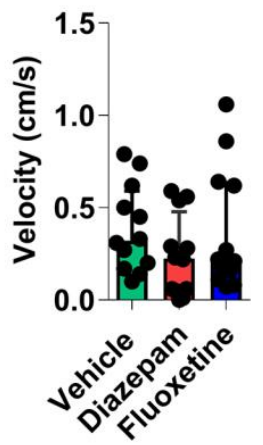

B

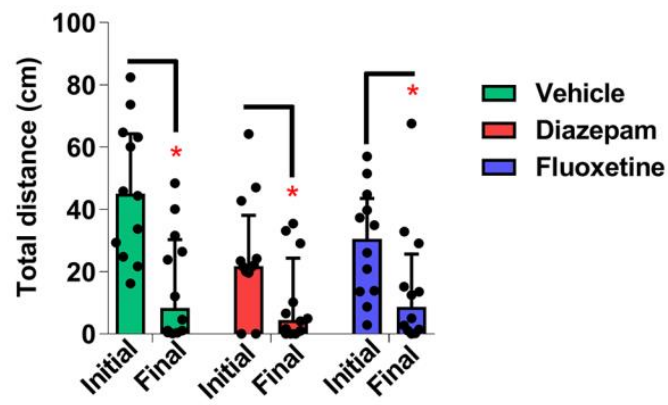

E
$F$
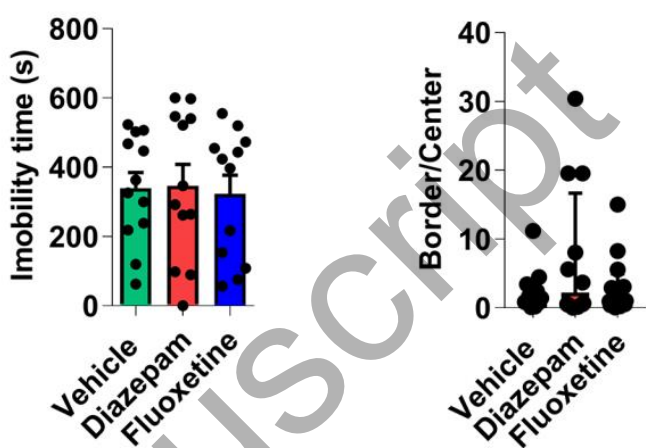

H

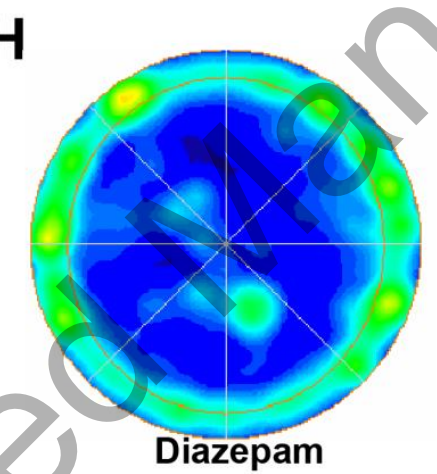

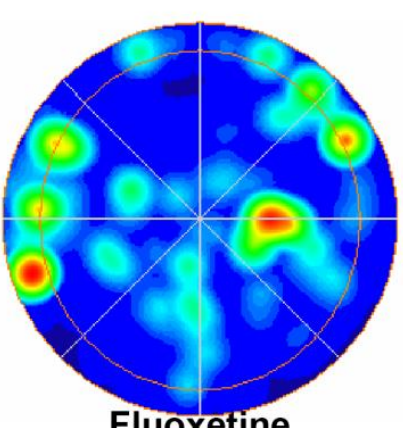

Fluoxetine 
A

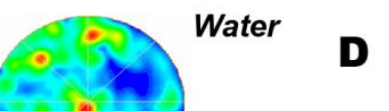

B

Vehicle

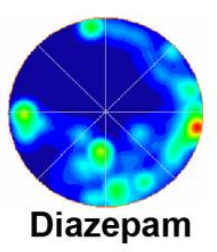

C

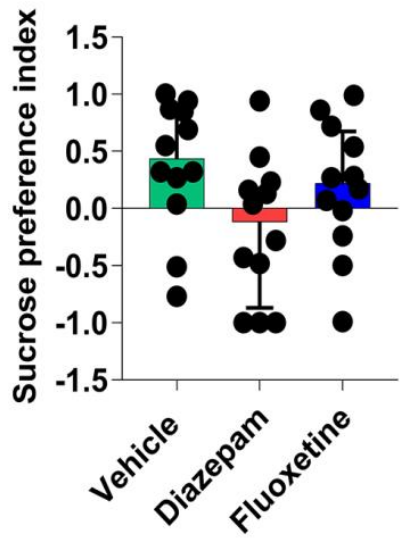

Fluoxetine 